\title{
AiMT
}

Advances in Military Technology

Vol. 12, No. 2 (2017), pp. 195-203

ISSN 1802-2308, eISSN 2533-4123

DOI 10.3849/aimt.01132

\section{Protection of Radio Electronic Devices and Electric Circuits from External Electromagnetic Influence}

\author{
O. Vorobiov* and O. Matsko \\ Logistic Department, National Defence University of Ukraine, Ukraine
}

The manuscript was received on 12 June 2016 and was accepted after revision for publication on 14 October 2017.

\begin{abstract}
:
Based on the existing methodologies of evaluating the effect of powerful electromagnetic pulse on susceptible objects, the following methodology has been developed. The main goal is to evaluate the effectiveness of protection of radio electronic devices and electric circuit equipment. Usually these devices and equipment are part of an industrial facility. Thus, to evaluate the effectiveness of protection, an Electromagnetic Influence (EMI) safety factor was taken as a system elements stability indicator, where the sustainability of the system is characterized by a minimal number of its elements. Sustainability of the system is estimated in stages; the algorithm implementation methodology is introduced with mathematical and physical expressions. These expressions define the degree of EMI energy neutralization that penetrates through intakes in the screens.
\end{abstract}

Keywords:

electromagnetic influence, effectiveness, electromagnetic energy, protection

\section{Introduction}

Finding solution to the problems related to the increasing firmness of Radio Electronic Facilities' (REF) electric networks is becoming more and more important. This can be due to further growth of control system computerization, increasing usage of REF and electric equipment. On the other hand, it is the recent expansion of natural cataclysms, accompanied by electrostatic and storm discharges along with researches on the creation of artificial sources of external EMI [1-3].

Alongside the mentioned dangers, protection method is being developed. It is based on screening, diverting destructive electromagnetic energy, its transformation into other types of energy and distribution by firmness of construction elements [4-7].

However, the article will not reveal the creation of new types of protection, but the estimation of its efficiency. Although the new methods and devices of protection do exist, there are no methodologies for the reliable efficiency estimation of the

\footnotetext{
* Corresponding author: Logistics Department, National Defence University of Ukraine named after Ivan Cherniahovskyi, Povitroflotsky prospekt 28, 03049 Kyiv, Ukraine.

Phone: +380 9774448 70, E mail: Oleg33377@ukr.net
} 
offered protection in certain cases. Therefore, there is an estimation methodology of electromagnetic impulse (EMP) effect on susceptible objects, but rarely there can be found any methodologies of protection efficiency estimation (e.g. creation of protection based on transformation of EMP destructive energy, that penetrates through intakes in the screens of susceptible objects).

\section{Basic Research Material Presentation}

The objective of this article is to develop a multipurpose estimation methodology of REF and electrical equipment circuits' protection efficiency based on the existent estimation methodologies of powerful EMP action on objects, sensible to these actions.

We will examine basic approaches to protection estimation according to the existing methodologies mentioned above. In this case, the factor of safety that is determined by the relation of a maximum possible applied current or intensity $U_{\sigma}$ to the applied one, which means a created EMI in given conditions $U_{\mathrm{e}}$, is accepted as an index of system elements' resistance to the EMI actions. The factor of safety is a logarithmic value that is measured in decibels, [dB] [8]

$$
K=\log \frac{U_{\sigma}}{U_{\mathrm{e}}},
$$

where $U_{\sigma}-$ the value of a maximum possible induced voltage;

$U_{\mathrm{e}}-$ the value of voltage of EMI created in these conditions.

The overall resistance of the system will be defined by the minimum safety factor value of all elements included, because different elements of the system can have different safety factor values.

The resistance of the system to the EMI action is estimated in the following sequence:

1. An expected EMI-situation is determined, mainly its parameters, defined by the electric $E=10^{5} \mathrm{~V} / \mathrm{m}$ and magnetic $H=250 \mathrm{Am}^{-1}$ field intensity accordingly, time period growth (for calculations we accept $t_{\mathrm{H}}=10^{-8} \mathrm{~s}$ and $t_{\mathrm{c}}=$ $10^{-6} \mathrm{~s}$ for slump of the electromagnetic field.

Then the function of the field slump will be as follows [6]:

$$
H_{0}(t)=250 \mathrm{e}^{-\frac{t}{10^{-6}}},
$$

where $H_{0}(t)$ - the magnetic field outside of screen in time $t$.

2. The electronic engineering system is divided into separate elements (areas), the analysis of setting of every element is conducted and base elements are distinguished. The work of the system will depend on these elements.

3. The responsiveness of apparatus and its elements to the EMI action is determined.

4. Possible values of currents and voltages of system elements are determined; the ones that are applied from the influence of EMI taking into account the weakening action of screen.

5. The safety factor of every system element and degree of system resistance to EMP is determined through the factor of safety by the formula (1). It is possible to consider the system degree of resistance as satisfactory, if $K \geq 40 \mathrm{~dB}$. 
6. The results of calculations are analyzed, estimated and the conclusion is drawn. It should specify the degree of system resistance to the EMI actions; the most vulnerable elements of the system; necessary organizational and engineering technical actions to increase the firmness of the sensitive elements and the system overall, taking financial viability into account.

The mentioned methodology [6] allows to estimate the force of external EMI acting on the susceptible system elements. However, it does not account completely for how the destructive energy of EMP penetrating through intakes in the screens is neutralized. Neither the influence of structural features of technological defects in the screens, the degree of destructive energy neutralization and its comparison to maximum resistance of REF is taken into consideration. It means that it does not completely take into account the aspects of protection efficiency estimation that is proposed.

In order to remove these defects and to increase the range of methodology of application in the third stage of its implementation, it is suggested to extend its possibilities by comparing data in relation to the maximum values of voltage and currents while the work of the system is not violated.

In addition to the algorithm of methodology implementation, it is suggested to add mathematical and physical expressions that define the degree of EMP energy neutralization that penetrates through intakes in screens. It will allow us to compare the efficiency of offered protection (screening, grounding, transformation and conversion of EMP energy).

In the future, the methodology should consider the efficiency of concrete screens and geometrical descriptions of openings (entrances) in screens, from the point of view of their influence on penetration of destructive EMP energy.

For this purpose, it is necessary to find the maximal value of magnetic field that passes through a screen (inside a screen).

For a spherical screen it equals [7]:

$$
H_{i \max }=\frac{R_{\mathrm{S}}}{L_{\mathrm{S}}} \int_{T_{1}}^{T_{2}} H_{0}(t) \mathrm{d} t,
$$

where $H_{0}(t)$ - the magnetic field outside a screen, equals:

$$
H_{0}(t)=H_{\max } \mathrm{e}^{-\frac{t}{t_{1}}}=250 \mathrm{e}^{-\frac{t}{10^{-6}}}
$$

where $H_{\max }$ - the maximal value of the external field, [A/m];

$t$ - the current time, [s];

$t_{1}$ - the time of the field slump $e$ times, [s];

$T_{2}-T_{1}-$ the pulse period, [s];

$R_{\mathrm{S}}$ - the equivalent successive resistance of screen, $[\Omega]$;

$L_{\mathrm{S}}-$ the equivalent successive inductance of screen, $[\mathrm{H}]$.

Then we will determine the value of time of the field growth inside a screen by the formula:

$$
t_{\mathrm{H}}=\left(\frac{d}{2.7}\right)^{2} \pi \sigma \mu,
$$

where $\mu$ - the magnetic permeability of the screen wall, $\left[\mathrm{Hm}^{-1}\right]$;

$d$ - the thickness of the screen wall, [m];

$\sigma-$ the conductivity of the screen material, $\left[\Omega^{-1} \mathrm{~m}^{-1}\right]$. 
Then we define the maximum intensity of magnetic field that penetrates (derivative from the intensity of magnetic field that penetrates) by the formula:

$$
H=\frac{H_{i \max }}{t_{\mathrm{H}}},
$$

After that we calculate the intensity in the system elements inside the screen. Then we find the electromotive force of application by the formula [7]:

$$
U_{e}=\mu_{0} H(t) S,
$$

where $S$ - the equivalent area of the inducing network (an area of equivalent coil for inducing that influences the sensitive elements), $\left[\mathrm{m}^{2}\right]$.

Let's examine the influence of defects in the screens. In particular, the openings substantially diminish a capacity for screening of electric field. The penetration of the opening in an endless plate is proportional to the cubed value of opening radius and it diminishes as a cubed value of the distance from the wall. This is applicable to an approximately round and small opening in comparison to a wavelength.

These correlations are presented in spherical coordinates [8]

$$
\begin{gathered}
E_{\mathrm{r}}=\frac{2 E_{0} R^{3}}{3 \pi r^{3}} \cos \theta, \\
E_{\theta}=\frac{E_{0} R^{3}}{3 \pi r^{3}} \sin \theta,
\end{gathered}
$$

where $r$ - the opening radius;

$E_{0}$ - the external field which is perpendicular to the wall;

$E_{\mathrm{r}}, E_{\theta}-$ the components of the vector of the external field in the distance $r$ from the opening;

$\theta$ - an angle between this direction and a perpendicular that passes through the centre of opening.

In case of small opening in a screen with solid walls, the magnetic field penetrates in the same way as the electric field. If an opening has a middle radius $R$ and a chamber is large, then the fields that are in a direct proximity to the openings are possible to be calculated using the following formulas [10]:

$$
\begin{gathered}
H_{\mathrm{r}}=\frac{4 R^{3} H_{0} \sin \varphi \sin \theta}{3 \pi r^{3}}, \\
H_{\varphi}=\frac{2 R^{3}}{3 \pi r^{3}} H_{0}, \\
H_{\theta}=\frac{2 R^{3}}{3 \pi r^{3}} H_{0} \sin \varphi \cos \theta,
\end{gathered}
$$

where $H_{0}$ - the tangent of the magnetic field out of the chamber;

$r$ - the distance is from the centre of opening to the point of measuring that is examined;

$\varphi, \theta$ - accordingly the azimuth angle in relation to $H_{0}$ and polar angle in relation to a normal to opening.

In addition to that, while effecting elements that conduct current, it is necessary to take into account that EMP has a horizontal and vertical component; that is why it is 
necessary to determine the value of electric field voltage which is applied both on vertical and horizontal areas of lines. The vertical component exceeds the horizontal component ten times and depending on the distance from a source of the external EMV, it is defined by the Eq. (13) and Eq. (14) for horizontal component [11, 12],

$$
\begin{gathered}
E_{\mathrm{V}}=5 \times 10^{3} \frac{(1+2 R)}{R^{3}} \log 14.5 q, \\
E_{\mathrm{H}}=10 \frac{(1+2 R)}{R^{3}} \log 14.5 q
\end{gathered}
$$

where $R$ - the distance from a source of external EMI, [m];

$q$ - the power of a source of external EMI, [W].

When calculating the external sources of EMI over the Earth's surface, the vertical component equals Eq. (15), horizontal component Eq. (16),

$$
\begin{gathered}
E_{\mathrm{V}}=5 \times 10^{3} \cdot K \frac{(1+2 R)}{R^{3}} \log 14.5 q, \\
E_{\mathrm{H}}=10 \cdot K \frac{(1+2 R)}{R^{3}} \log 14.5 q,
\end{gathered}
$$

where $K-$ the coefficient of asymmetry.

Finally, the voltage is defined that is applied to electronic components and electrical networks by the following formulas [11, 12]: in vertical sections of lines and elements that carry intensity Eq. (17) and Eq. (18) in horizontal

$$
\begin{aligned}
& U_{\mathrm{V}}=\frac{E_{\mathrm{V}} \cdot l}{\eta}, \\
& U_{\mathrm{H}}=\frac{E_{\mathrm{H}} \cdot l}{\eta},
\end{aligned}
$$

where $l$ - the length of wire, [m];

$\eta$ - the line shielding coefficient (element).

After that, the values are compared with the maximum allowed, and the effectiveness of the proposed protection is evaluated.

The suggested method can be illustrated by the example of the efficiency estimation of REF protection. Experimental research consisted of ten applications of certain EMI source on REF (sensitive semiconductor devices) that were in the screened chamber in condition of intake protection absence in the screen.

The results of the research of a semiconductor devices' damage dependency on the parameters of EMI are listed in Tab. 1 and 2.

The complete damage, degradation and partial damage parameters were taken as the degree of REF damage. The power of impulse, frequency of the impulses, pulse width and intensity of electric and magnetic components of the electromagnetic field were taken as parameters of EMI.

These results of the research allow to conclude that the protection buffer is able to protect semiconductor devices from the EMI with certain parameters to provide them with conditions for reliable functioning.

The next step of the method implementation is the detection of ranges from a source where the intensity of EMI energy is equal to similar EMI of experimental source. For this purpose the following actions were performed: 
Tab. 1 Dependency of semiconductor devices damages from the parameters of striking electromagnetic impulse

\begin{tabular}{|c|c|c|c|c|c|c|c|c|}
\hline \multirow[b]{2}{*}{ No. } & \multicolumn{5}{|c|}{ Parameters of striking electromagnetic impulse } & \multicolumn{3}{|c|}{$\begin{array}{l}\text { Degree of semiconduc- } \\
\text { tor devices damages }\end{array}$} \\
\hline & 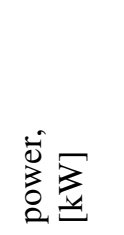 & 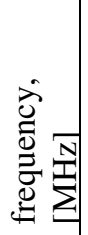 & 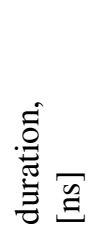 & 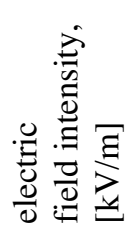 & 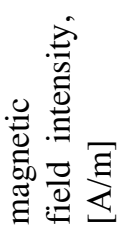 & 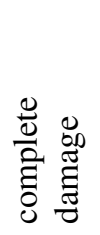 & 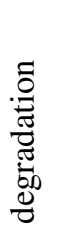 & 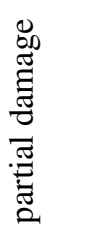 \\
\hline 1. & $1 \times 10^{-1}$ & 30 & 60 & 50 & 240 & yes & no & no \\
\hline 2. & $5 \times 10^{-2}$ & 30 & 60 & 45 & 230 & yes & no & no \\
\hline 3. & $1 \times 10^{-2}$ & 30 & 60 & 40 & 220 & yes & no & no \\
\hline 4. & $8 \times 10^{-3}$ & 30 & 60 & 35 & 210 & no & yes & no \\
\hline 5. & $4 \times 10^{-3}$ & 30 & 60 & 30 & 200 & no & yes & no \\
\hline 6. & $1 \times 10^{-3}$ & 30 & 60 & 25 & 190 & no & yes & no \\
\hline 7. & $7 \times 10^{-4}$ & 30 & 60 & 20 & 180 & no & no & yes \\
\hline 8. & $3 \times 10^{-4}$ & 30 & 60 & 15 & 170 & no & no & yes \\
\hline 9. & $1 \times 10^{-4}$ & 30 & 60 & 10 & 160 & no & no & yes \\
\hline 10. & $1 \times 10^{-5}$ & 30 & 60 & 5 & 150 & no & no & no \\
\hline
\end{tabular}

1. An intensity of power fluence $P_{\mathrm{f}}$ of EMI energy which is expected on intakes of the screen which appears from the source of EMI.

It is known that striking energy of the electromagnetic field can be passed by Hertzian waves which have a form of a flat wave, in the distance which is large enough. Therefore, there is an expedient formula for calculating power fluence:

$$
P_{\mathrm{f}}=\frac{P_{i} \cdot \mathrm{D}_{\mathrm{ad}}}{4 \pi \cdot R^{2}},
$$

where $P_{i}$ - is the power of Hertzian waves' vibrations that are emanated and accepted $1 \mathrm{GW} ; \mathrm{D}_{\mathrm{ad}}$ - is the coefficient of antenna directed action to the source of EMI, having frequency $100 \mathrm{MHz}$ acceptable range is $5 \div 10$, for $10 \mathrm{GHz}-50 \div 70 ; R$ - is the distance from the source of EMI to the object.

2. Using the analytical dependency, a graph displaying the dependency of power fluence $P_{\mathrm{f}}$ from the distance $R$ on the source of EMI at a pulse width $60 \mathrm{~ns}$ can be built (Fig. 1).

3. The distances where the damages of REF can occur are determined using the resulting graph.

4. In the final step of the research, the estimation of protection efficiency is performed. For this purpose the amount of EMI energy which is possible to convert into other kinds of energy using the protection buffers for intake holes in screens has to be calculated by Eq. $(8) \div(12)$. 
Tab. 2 Dependency of semiconductor devices damages from the parameters of striking electromagnetic impulse in condition of protective buffer application

\begin{tabular}{|c|c|c|c|c|c|c|c|c|}
\hline \multirow[b]{2}{*}{ No. } & \multicolumn{5}{|c|}{ Parameters of striking electromagnetic impulse } & \multicolumn{3}{|c|}{$\begin{array}{c}\text { Degree of semiconduc } \\
\text { tor devices damages }\end{array}$} \\
\hline & 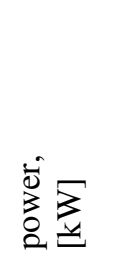 & 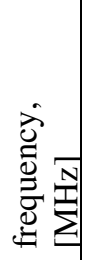 & 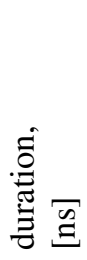 & 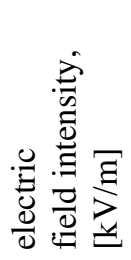 & 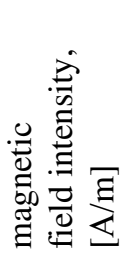 & 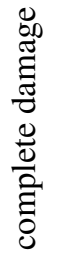 & 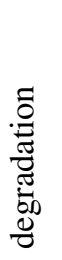 & 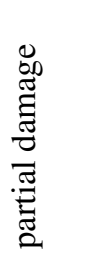 \\
\hline 1. & $1 \times 10^{-1}$ & 30 & 60 & 50 & 240 & no & no & yes \\
\hline 2. & $5 \times 10^{-2}$ & 30 & 60 & 45 & 230 & no & no & no \\
\hline 3. & $1 \times 10^{-2}$ & 30 & 60 & 40 & 220 & no & no & no \\
\hline 4. & $8 \times 10^{-3}$ & 30 & 60 & 35 & 210 & no & no & no \\
\hline 5. & $4 \times 10^{-3}$ & 30 & 60 & 30 & 200 & no & no & no \\
\hline 6. & $1 \times 10^{-3}$ & 30 & 60 & 25 & 190 & no & no & no \\
\hline 7. & $7 \times 10^{-4}$ & 30 & 60 & 20 & 180 & no & no & no \\
\hline 8. & $3 \times 10^{-4}$ & 30 & 60 & 15 & 170 & no & no & no \\
\hline 9. & $1 \times 10^{-4}$ & 30 & 60 & 10 & 160 & no & no & no \\
\hline 10. & $1 \times 10^{-5}$ & 30 & 60 & 5 & 150 & no & no & no \\
\hline
\end{tabular}

In the future, for the direct protection efficiency estimation, all equipment depending on firmness to EMI can be divided into five groups:

1) devices of commutation and electrical engineering devices (maximum electromagnetic firmness is $103 \div 109 \mathrm{~kW}$ );

2) electric vacuum devices (maximum electromagnetic firmness is $10 \div 102 \mathrm{~kW})$

3) thermic elements (maximum electromagnetic firmness is $1 \div 10 \mathrm{~kW}$ );

4) electrochemical and photosensitive devices (maximum electromagnetic firmness is $10^{-2} \div 10^{-1} \mathrm{~kW}$ );

5) semiconductor devices (maximum electromagnetic firmness is $10^{-3} \div 10^{-2} \mathrm{~kW}$ ).

It was discovered that the effective action of EMI source starts from $15 \div 20 \mathrm{~m}$ from the object of protection. The distance that is safe for REF is determined by the resistance of its different components. By analysing Fig. 1, it is possible to note that the influence of EMI source of certain power on REF of most sensitiveness $\left(10^{-2} \mathrm{~kW}\right)$ is $10000 \mathrm{~m}$, for the least sensitiveness $(104 \mathrm{~kW})$ it is $100 \mathrm{~m}$.

The efficiency of protection is determined as a correlation of the object distance from the EMI source, where maximum electromagnetic interoperability between separate REF is yet provided for the real protection. 


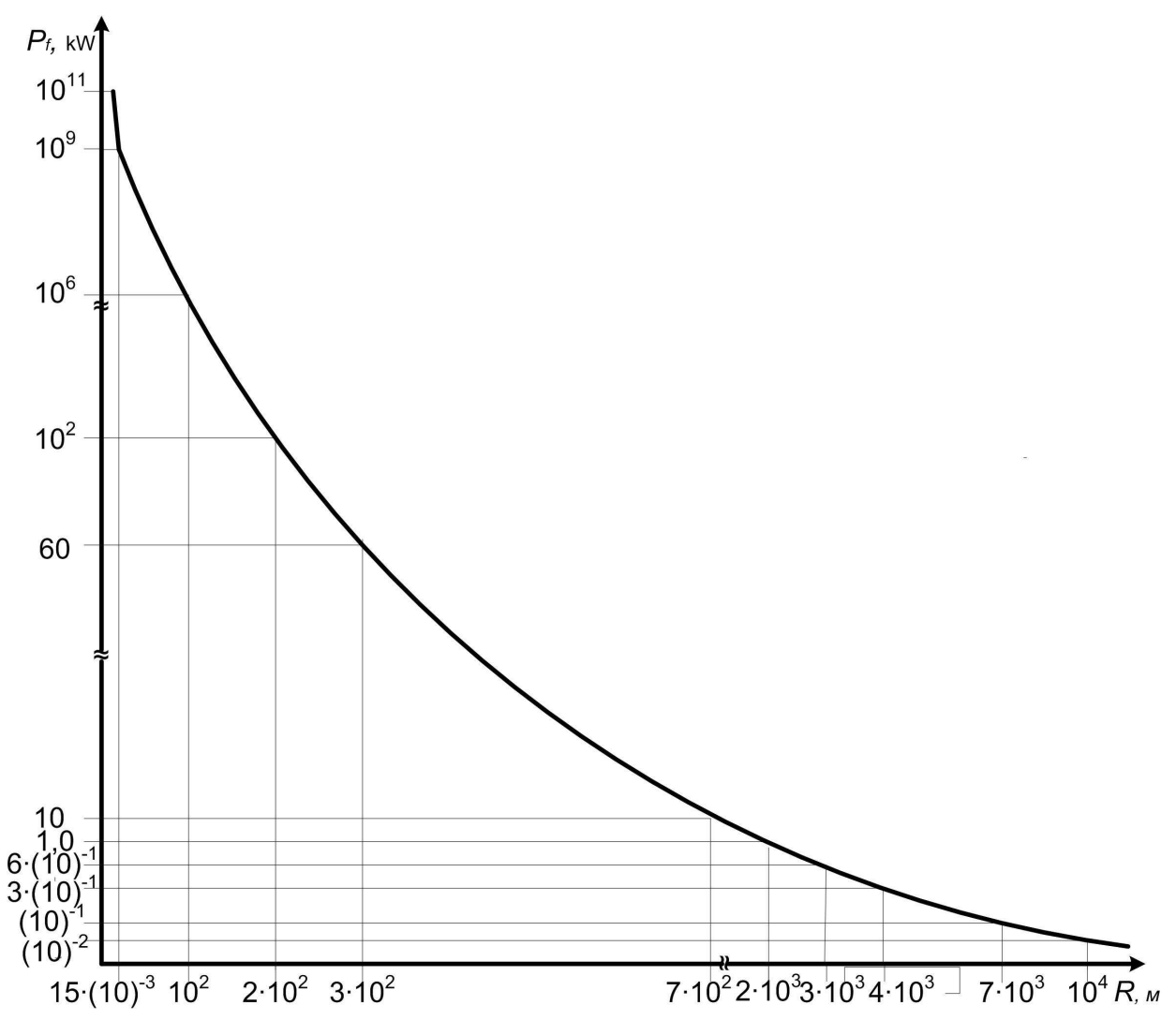

Fig. 1. Dependence offluence power $P_{f}$ from the distance $R$ to the source of electromagnetic influence at a pulse width $60 \mathrm{~ns}$

\section{Conclusions}

To sum up, suggested evaluation method of the effectiveness of REF and electric circuits' protection that are part of the industrial and technical objects from the external EMI is not similar to the existing methods of evaluation the EMP effect. Unlike them, it allows to consider the physical features of vulnerable system objects protection to the fullest. The parameters that characterize the degree of EMP energy neutralization penetrating through openings (entrances) in the screens are taken into account either. This allows to compare the performance of a particular type of protection proposed (shielding, grounding, transformation and EMP energy conversion etc.).

\section{Directions of Further Investigations}

It is proposed to develop evaluation methods for each type of protection for various industrial and technical facilities in the future. 


\section{References}

[1] KRAVCHENKO, V.I. Lightning. Electromagnetic Factors and their Destructive Effects on the Technical Means (in Russian). 2010, 292 p.

[2] BEGICH, N. Nikola Tesla and his Diabolical Weapons. Moscow: Yauza, Eksmo, 2008, $384 \mathrm{p}$.

[3] BERDYSHEV, A.V. Experimental Research on the Effects of Microwave Pulses on Integrated Circuits Containing Radio-Electronic Devices (in Russian). Radio Engineering, 2000, no. 6, p. 85-88.

[4] BOGUSH, V.A., BORBOTKO, T.V. and GUSINSKY, A.V. Electromagnetic Radiation. Methods and Means of Protection. Munich: Bestprint, 2003, 406 p.

[5] BALUK, N.V. The Powerful Electromagnetic Pulse: Effects on Electronic Means and Methods of Protection (in Russian). Moscow: Group of IDT, 2007, 478 p.

[6] MIKHAILOV, V.A. Ensuring Stability of the Onboard Digital Computers to the Effects of Uultrashort Electromagnetic Pulses. [PhD Thesis]. Moscow, 2009, 24 p.

[7] MYROVA, L.O. Ensuring Stability of Communications Equipment to the Ionizing and Electromagnetic Radiation. Moscow: Radio and Communication, $2^{\text {nd }}$ ed., 1988, $296 \mathrm{p}$.

[8] EYGENBORT, V.M. Converters Kind of Signal Energy in Systems Management Automation. Moscow: Energy, 1970, 272 p.

[9] DEMIDENKO, G.P., KUZMENKO, E.P., ORLOV, P.P. et al. Protection of National Economy by Weapons of Mass Destruction. Kyiv: Vishcha shkola, $2^{\text {nd }}$ ed., 1989, 287 p.

[10] RICKETTS, L.U. Electromagnetic Pulse and Methods of Protection. Moscow: Atomizdat, 1979, $327 \mathrm{p}$.

[11] NOVGORODTSEV, A.B. The Calculation of Electric and Magnetic Fields. Al: LPI, 1975, $80 \mathrm{p}$.

[12] TOZONI, O.V. Mathematical Models for the Calculation of Electric and Magnetic Fields (in Ukrainian). Kyiv: Naukova Dumka, 1986, 304 p. 